\title{
DEAMBULAÇÃO APÓS O USO DE APARELHOS DE FIXAÇÃO EXTERNA OU PINOS INTRAMEDULARES NA TÍBIA DE CÃES SADIOS ${ }^{1}$
}

\author{
FUNCTIONAL RECOVERY AFTER USE OF EXTERNAL SKELETAL \\ FIXATION OR INTRAMEDULLARY PINS IN HEALTHY DOGS
}

\author{
Murilo Nogueira dos Santos Junior ${ }^{2}$ João Eduardo Schossler ${ }^{3}$
}

RESUMO

Avalia-se, clínica e comparativamente, o aparelho de fixação externa e pinos intramedulares, quanto ao tempo de recuperação das funções normais e deambulação de cães sadios. Foram utilizados 16 cães sadios separados em dois grupos experimentais de oito animais. No primeiro grupo, os cães foram submetidos à aplicação de um aparelho de fixação externa e, no segundo grupo, submetidos à inserção de dois pinos intramedulares na tíbia esquerda. Os aparelhos de fixação externa foram retirados após 30 dias de avaliação clínica. Observou-se que os cães com fixadores externos tiveram retorno funcional em média de 17,3 dias, enquanto os submetidos à inserção intramedular tiveram retorno aos 4,2 dias. Com esses resultados, conclui-se que o uso de pinos intramedulares tem menor interferência na deambulação em relação ao aparelho de fixação externa.

Palavras-chave: fratura de tíbia, pinos intramedulares de Steinmann.

\section{SUMMARY}

In this study we evaluate the contribution of external skeletal fixation devices and intramedullary pins in the functional recovery of healthy dogs. Sixteen healthy dogs have been divided into two experimental groups of 8 dogs each. The first group was submitted to external skeletal fixation devices and the second group was submitted to intramedullary pins in the left Tibia. The external skeletal fixation was taken out by the thirtieth day of the clinical evaluation. The dogs that were submitted to external fixation devices had functional recovery of the studied limb of 17.3 days (in average), while those submitted to intramedullary pins had the functional recovery of the limb in 4.2 days. We concluded that the intramedullary pins have less interference in the functional recovery of the affected limb when compared to the external skeletal fixation devices.
Key words: dogs, tibial fractures, external skeletal fixation, intramedullary pins, functional recovery.

\section{INTRODUÇÃO}

A escolha do método de fixação baseia-se no tipo e localização da fratura, tamanho e idade do animal, número de ossos envolvidos e viabilidade dos tecidos moles da região. Muitos métodos de fixação são propostos para o tratamento de fraturas diafisárias de tíbia. A fixação por pinos intramedulares é um dos métodos de fixação interna mais utilizados, provavelmente pelo seu baixo custo, facilidade de aplicação e pela vantagem biomecânica de resistir a cargas de encurvamento em qualquer direção (DE YOUNG \& PROBST, 1993). O pino de Steinmann, por ser colocado no canal medular, é o implante mais utilizado em Medicina Veterinária e o menos sofisticado. Sua força está relacionada com seu diâmetro e sua capacidade de resistir à mobilidade do fragmento fraturado através do seu contato com o endósteo. Quando colocado, deve estar em estrito contato com o córtex medular de maneira a possibilitar alguma resistência às forças rotacionais. Para aumentar esta estabilidade, podem ser utilizados dois ou mais pinos. (NUNAMAKER \& NEWTON, 1985).

Outro método utilizado é a redução da fratura por fixadores externos, que consiste na inserção percutânea de pinos, que atravessam a pele,

\footnotetext{
${ }^{1}$ Dissertação de mestrado apresentada pelo primeiro autor ao Programa de Pós-graduação em Medicina Veterinária, Centro de Ciências Rurais (CCR), Universidade Federal de Santa Maria (UFSM), RS.

${ }^{2}$ Médico Veterinário, Mestre, Hospital de Clínicas Veterinárias, CCR, UFSM, 97105-900, Santa Maria, RS. E-mail: Murilion@hotmail.com. Autor para correspondência.

${ }^{3}$ Médico Veterinário, Doutor, Professor adjunto do Departamento de Clínica de Pequenos Animais, CCR, UFSM. Recebido para publicação em 16.11.00. Aprovado em 04.07.01
} 
tecidos moles e ambas corticais do osso, sendo fixados externamente por hastes ou barras de natureza metálica ou de acrílico autopolimerizante. A estabilização dos fragmentos ósseos, sem causar danos excessivos aos tecidos moles adjacentes e à vascularização óssea, além de evitar a necessidade de implantes no local da fratura ou imobilização das articulações, são vantagens do método que é indicado na redução de fraturas cominutivas ou expostas, em que há maior risco de infecção, comprometimento vascular e que necessitam de longo tempo de imobilização. Sua segurança e versatilidade permitem que o método seja empregado naqueles casos em que outros métodos não foram eficazes (EGGER,1991).

Tendo em vista que, em muitos casos de fraturas diafisárias de tíbia, é possível a aplicação, tanto de um fixador externo quanto a inserção de pinos intramedulares, para a osteossíntese dos fragmentos, avaliou-se, clínica e comparativamente, os dois métodos, quanto ao tempo de recuperação das funções normais e deambulação do membro operado.

\section{MATERIAL E MÉTODOS}

Foram utilizados 16 cães machos e fêmeas, sem raça definida, de idades variadas, adultos, com peso corpóreo entre 9 e $20 \mathrm{~kg}$, clinicamente sadios e todos provenientes do Biotério Central da Universidade Federal de Santa Maria, separados em dois grupos de oito animais. Num período de adaptação de sete dias, eles foram submetidos a exames clínicos e desverminação com Pamoato de Pirantel na dose de $10 \mathrm{mg} / \mathrm{kg}$, por via oral e avaliados radiologicamente, antes da cirurgia, para a verificação das estruturas anatômicas

O protocolo anestésico, para todos os animais, constituiu-se de medicação pré-anestésica à base de acepromazina $^{\mathrm{a}}(0,2 \mathrm{mg} / \mathrm{kg})$ por via intramuscular, indução anestésica com tiopental sódico $^{\mathrm{b}}(10 \mathrm{mg} / \mathrm{kg})$, por via endovenosa e a manutenção anestésica com halotano ${ }^{c}$ e oxigênio em circuito anestésico semifechado através de sonda endotraqueal. Trinta minutos antes do procedimento cirúrgico foi administrada ampicilina sódica ${ }^{\mathrm{e}}$ $(20 \mathrm{mg} / \mathrm{kg})$ por via intravenosa, como antibiótico profilático.

Nos animais do grupo I, quatro pinos de Steinmann, com as pontas trifacetadas, possuindo um diâmetro de aproximadamente $20 \%$ do diâmetro do osso, foram introduzidos de maneira a atravessar a pele, tecidos moles, cortical óssea, medula óssea, cortical oposta e tecidos moles, fazendo com que os mesmos emergissem no lado oposto do membro.
Para a introdução dos pinos, foi utilizado o introdutor manual tipo Jacob.

Inicialmente foram introduzidos os pinos distal e proximal em relação à diáfise do osso em ângulo de 90 graus, seguindo-se a introdução dos outros dois pinos com uma angulação aproximada de 70 graus em relação ao osso. As extremidades dos pinos foram envergados paralelamente ao eixo longitudinal do osso, onde se colocou uma barra de acrílico autopolimerizável ${ }^{\mathrm{d}}$, confeccionada manualmente, que envolvia e unia as extremidades dos pinos, em ambos os lados, situado a $2 \mathrm{~cm}$ da pele. Aplicou-se então uma bandagem com atadura de crepom, e foi administrado o agente antiinflamatório fluximin meglumine $\mathrm{e}^{\mathrm{e}}(1,1 \mathrm{mg} / \mathrm{kg})$ por três dias, por via subcutânea. Imediatamente após a cirurgia, os animais eram encaminhados ao setor de radiologia para reavaliação.

Os curativos externos foram feitos diariamente com pomada antimicrobiana à base de nitrofurazona ${ }^{\mathrm{f}}$, seguida de colocação de bandagem com atadura de crepom ao redor do aparelho até a retirada do mesmo, que ocorreu 30 dias após a cirurgia. Durante este período, os animais foram avaliados e suas manifestações clínicas registradas em ficha apropriada. Para a retirada do aparelho, os animais foram submetidos à sedação com cloridato de xilazina ${ }^{\mathrm{g}} 2 \%(1 \mathrm{mg} / \mathrm{kg})$ por via intravenosa, antisepsia da área e dos pinos com álcool iodado, sendo os pinos cortados próximo a sua inserção, em ambos os lados e retirados com auxílio de um alicate ortopédico esterilizado. $\mathrm{Na}$ seqüência, foi feita a limpeza dos orifícios com solução salina e após aplicação tópica de tintura de timerosal ${ }^{\mathrm{h}}$. Após esta retirada dos pinos, os animais foram novamente radiografados.

No grupo II, os oito cães foram submetidos à inserção de pinos intramedulares através da crista tibial. Após a anti-sepsia da área com os animais posicionados em decúbito dorsal, foi feita a inserção de maneira normógrada, de dois pinos intramedulares de Steinmann com auxílio do introdutor manual tipo Jacob. A introdução foi realizada em duas etapas: a introdução completa do primeiro pino a fim de permitir a introdução do segundo. Este primeiro pino foi inserido através da pele e ao longo da borda medial do ligamento patelar, penetrando a extremidade proximal da tíbia em um ponto médio entre a tuberosidade da tíbia e o ligamento colateral medial. Após o pino chegar à extremidade óssea distal, foi recuado dois milimetros, cortado e, com auxílio de um impactador de pinos e um martelo cirúrgico, o mesmo foi novamente conduzido à profundidade original.

Foi feito um ponto isolado tipo Wolf no local da incisão de colocação dos pinos, não sendo necessária a colocação de bandagens. 
O pós-operatório imediato foi o mesmo aplicado aos animais do grupo I, e o membro operado foi radiografado para avaliação durante um período de 30 dias. Todos os animais foram avaliados em suas manifestações clínicas em cinco graus conforme o citado por TUDURY \& RAISER, (1985) para verificação do uso funcional do membro (Tabela 1). Os pinos não foram retirados, pois se optou pelo sepultamento dos mesmos.

\section{RESULTADOS}

No grupo I, dos cães que foram submetidos ao aparelho de fixação externa, três animais obtiveram retorno funcional do membro no $8^{\circ}$ dia de pós operatório, um no segundo dia e outro no $23^{\circ}$ dia. Três cães não obtiveram retorno funcional do membro (Figura 1A). O cão número 4 teve uso ou apoios infreqüentes, foi sacrificado e, na necropsia, apresentou lesão no tendão do músculo extensor lateral dos dedos; o de número 8 teve apoio normal em estação e não claudicava ao andar, mas elevava ao correr, e não apresentava lesões no local de inserção dos pinos; o cão de número 3 apresentou afrouxamento de um pino no $12^{\circ}$ dia, devido a um processo infeccioso que se instalou. No local de inserção deste pino, a pele se apresentava com um pequeno aumento de volume com hiperemia e com uma discreta secreção serosa ao redor do orifício.

Nos animais do grupo II, submetidos à inserção de pinos intramedulares (Figura 1B), quatro cães obtiveram retorno funcional no segundo dia, dois no quinto, um no sétimo e outro no décimo dia.

\section{DISCUSSÃO}

O breve retorno funcional de um membro fraturado é o objetivo da redução de fraturas, segundo DE YOUNG \& PROBST (1993). Daí a

Tabela 1 - Características clínicas dos cinco graus de claudicação utilizados para apreciar a recuperação do uso funcional do membro nos cães submetidos à inserção de pinos intramedulares ou aparelho de fixação externa na tíbia.

\begin{tabular}{|c|c|}
\hline GRAUS & CARACTERÍSTICAS \\
\hline I & Não usa nem apoia o membro \\
\hline II & $\begin{array}{l}\text { Uso e apoio infreqüente do membro na estação e no caminhar. } \\
\text { Não suporta peso na extremidade afetada, elevando-a ao correr }\end{array}$ \\
\hline III & $\begin{array}{l}\text { Claudicante uso do membro na estação e no caminhar. Parcial } \\
\text { suporte do peso na extremidade, elevando-a ao correr }\end{array}$ \\
\hline IV & Caminha sem claudicar. Normal na estação. Claudica ao correr \\
\hline $\mathrm{V}$ & Uso funcional do membro \\
\hline
\end{tabular}

Fonte: TUDURY\& RAISER (1985). realização do experimento para avaliar a influência dos métodos de fixação externa ou do uso de pinos intramedulares na tíbia de cães sadios, entre os inúmeros métodos existentes para a redução de fraturas de ossos longos, em cães.

No grupo I, optou-se pela utilização do aparelho do tipo II, pois segundo EGGER (1993), HULSE \& HYMAN (1991) e LEWIS \& BLOOMBERG (1994), esta configuração oferece maior resistência que o aparelho tipo I, além de possibilitar um maior tempo de permanência no animal. Com a utilização de pinos com diâmetro de aproximadamente $20 \%$ do diâmetro do osso (HARARI, 1996), observaram-se menor claudicação e um retorno mais rápido do uso do membro. Isto se deve à maior interface pino-osso, garantindo uma melhor distribuição das forças atuantes e evitando o excesso de carga localizada (HARARI, 1996). Já o uso de implantes com diâmetro exagerado pode correr o risco de se fragilizar o osso, favorecer ou provocar fraturas, segundo NUNAMAKER \& NEWTON, (1985).

Os pinos distal e proximal foram os primeiros a serem introduzidos, seguindo orientação de HARARI (1996), que enfatizou ser esta uma maneira segura de observar a anatomia do membro e ainda planejar o posicionamento e a distância entre os demais pinos. Optou-se pela angulação de 70 graus mantida entre os pinos e o eixo longitudinal do osso já, segundo JOHNSON \& FISCHER (1983), aumenta a resistência do aparelho e evita o deslizamento latero-medial do mesmo; com isto a utilização de pinos lisos só causou afrouxamento do aparelho de fixação externa em um animal ao contrário do que citou TOOMBS (1996), que indicou o uso exclusivo de pinos rosqueados para esta técnica.

A utilização do introdutor manual tipo Jacob's não causou aquecimento e necrose óssea, cuidados que devem ser observados segundo SPIEGEL \& VANDERSCHILDEN (1983) e TOOMBS (1996). Entretanto, os mesmos autores relataram que a utilização deste tipo de introdutor pode favorecer o aparecimento de reação periostal ao redor dos pinos por estimulação osteoclástica, provocando afrouxamento dos pinos o que pode ter acontecido com um dos cães, embora o aparelho permanecesse estável mesmo com o processo infeccioso que se instalou. TOOMBS (1996) citou ser imprescindível a utilização do guia de broca para orientar a penetração do pino percutâneo, para que não ocorra afrouxamento precoce dos mesmos. Este fato não foi constatado, embora tenha se observado 


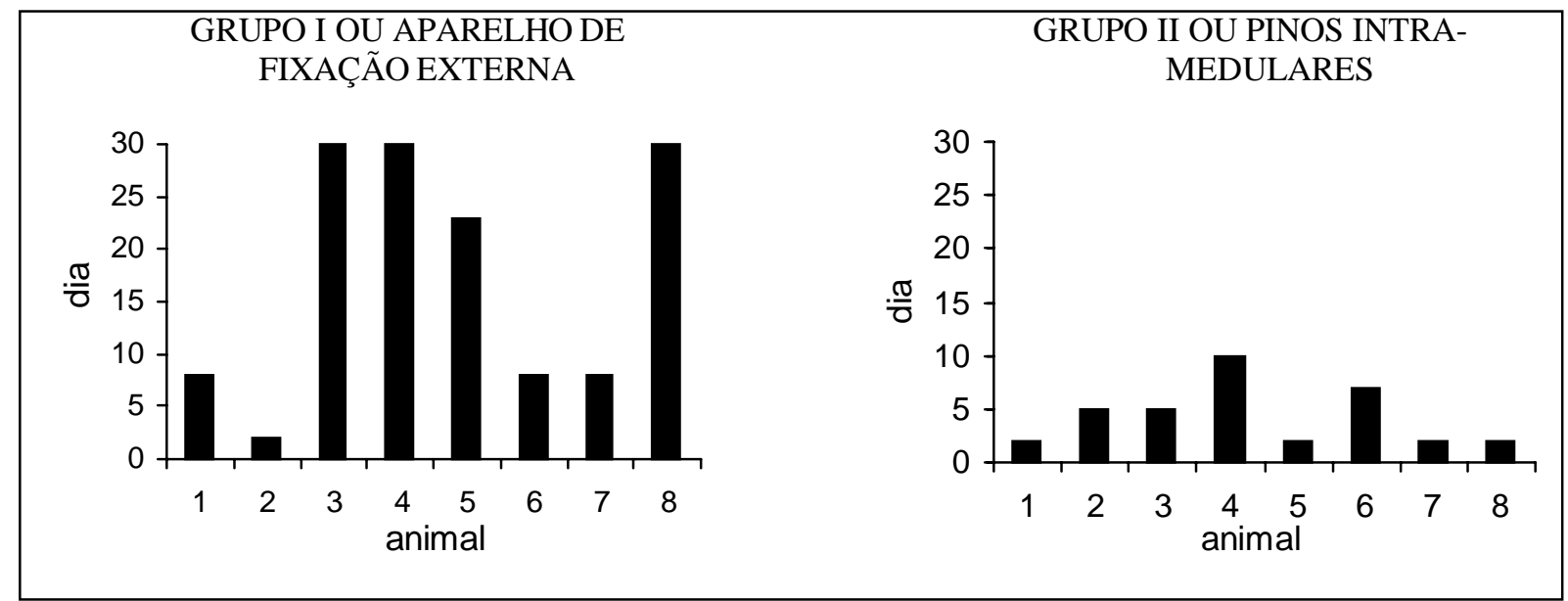

Figura 1 - Os gráficos representam os dias do retorno funcional dos membros operados dos animais do grupo I (aparelho de fixação externa), e do grupo II (pinos intramedulares).

um enovelamento dos tecidos moles ao redor dos pinos devido à falta de proteção do guia e da incisão e divulsão prévias, o que pode ter favorecido o aparecimento de exsudato purulento ao redor de alguns pinos.

A higienização dos pontos de inserção dos pinos na pele obedeceu às freqüências recomendadas por GREEN (1983) e SISK (1983) e demonstraram ser os intervalos adequados também para a observação do membro operado. A utilização de antiinflamatório parenteral, durante os primeiros três dias de pós-operatório, citada por SMITH (1993), foi eficiente para inibir o processo inflamatório, assim como o cuidado de manter a haste acrílica a dois centímetros de distância da pele do animal para evitar a compressão dos tecidos moles contra a mesma.

O metilmetacrilato, como haste externa, evidenciou algumas das vantagens enunciadas por OHASHI $\boldsymbol{e}$ t al. (1983), OBRASINSKI et al. (1991) e WILLER et al. (1991), como a versatilidade do material, facilidade de acesso às feridas para higienização e a característica radioluscente do material o que favorece na avaliação radiográfica.

Nos animais do grupo II, a técnica operatória foi a recomendada por BARON (1991), e, com ela, procurou-se obter pontos de apoio proximais e distais para os pinos. O canal medular teve ultrapassado o preenchimento de $70 \%$ conforme o descrito por SCHRADER (1991), com o objetivo de aumentar o contato do pino com o endósteo.

A introdução dos pinos por meio de um condutor de Jacob's, conforme recomendações de DE YOUNG \& PROBST, (1993), foi fundamental para a eficácia do método de inserção normógrada, por assegurar a precisão no momento de produzir a angulação necessária na produção do encurvamento dos pinos. O que não acontece com outros tipos de introdutores com os quais não se consegue obter a angulação necessária, e o pino transfixa a cortical óssea. O encurvamento do pino, quando posicionado no canal medular, foi fundamental para evitar essa transfixação, conforme relatou SCHRADER (1991) e a utilização de dois pinos foi vantajoso, pois foi possível o preenchimento do canal medular utilizando pinos de diâmetro menor e, por conseguinte, maior maleabilidade, permitindo assim encurvamento necessário. De acordo com DE YOUNG \& PROBST (1993), quanto maior o pino, maior a dificuldade de se obter o encurvamento.

A inserção dos pinos através da crista tibial não causou sinais de lesão articular, nem interferência condilar como preconizaram PARDO (1994) e DIXON (1994). Os animais deste experimento tinham estrutura óssea com canal medular retilíneo, o que talvez tornasse favoráveis os resultados obtidos.

Os pinos não foram retirados após os trinta dias de avaliação, pois no exame clínico e na análise radiográfica, verificou-se que os mesmos estavam sepultados na medular do osso. Este procedimento foi recomendado por BIERY (1985) e DE YOUNG \& PROBST(1993).

$\mathrm{O}$ uso de flumixin meglumine devido a suas ações antinflamatória e analgésica pode ter contribuído na regressão mais rápida das lesões decorrentes do ato cirúrgico e na redução do período de convalescência do animal, acelerando o retorno a deambulação. Não ocorreu atrofia muscular em nenhum dos animais, devido à recuperação precoce do apoio do membro operado. 
Deste experimento, pode-se concluir que houve nítida vantagem do método de introdução de pinos intramedulares sobre o método de fixação externa da tíbia no restabelecimento da deambulação fisiológica.

\section{FONTES DE AQUISIÇÃO}

${ }^{a}$ Acepran $1 \%$. Univet. S. A. Indústria Veterinária. São Paulo - SP. ${ }^{\mathrm{b}}$ Thionembutal. Abbott. Laboratório North Chicago. São Paulo SP.

${ }^{\mathrm{c}}$ Halotano. Hoerchst do Brasil. Química e Farmacêutica S. A . Suzano - SP.

${ }^{\mathrm{d} A m p i c i l i n a}$ Veterinária. Univet S.A. Indústria Veterinária São Paulo - SP.

${ }^{\mathrm{e} J e t}$ Acrílico. Acrílico autopolimerizante. Artigos Odontológicos Clássico. São Paulo -SP

${ }^{\mathrm{f}}$ Banamine.Schering-Plough Veterinária. Jacarepaguá. Rio de Janeiro - RJ.

${ }^{\mathrm{g}}$ Nitrofurazona. IFAL Indústria e Comércio de Produtos Farmacêuticos. Camaquã - RS.

${ }^{\mathrm{h}}$ Coopazine. Mallinckrodt Veterinária Ltda. Cotia - SP

'Timerosal Incolor. Ibiza Química Ltda. Guarulhos - SP

\section{REFERÊNCIAS BIBLIOGRÁFICAS}

BARON, M. L'enclouage centro-medullaire des fractures diaphysaires du juene en croissance. Pratique Medical et Chirurgicale de I'Animal de Compagnie, v.26, n.3, p.203209, 1991.

BIERY, D.N. Orthopedic radiografy. In: NUNAMAKER, D.M., NEWTON, C.D. Textbook of small animal orthopaedics. Philadelphia : Lippincott, 1985. Cap.7, p.133-142.

DE YOUNG, D.J., PROBST, C.W. Methods of internal fracture fixation. In: SLATTER, D. Textbook of small animal surgery. Philadelphia : Saunders, 1993. V.2. Cap.122, p.1610-1631.

DIXON, B.C. Effects on three intramedulary pinning techniques on proximal pin lacation and articular demage in canine tibia. Veterinary Surgery, v.22, p.273-283, 1994.

EGGER, E.L. Complications of sternal fixation. Veterinary Clinics of Norh America: Small Animal Practice, v.21, n.4, p.705-733, 1991 .

EGGER, E.L. External skeletal fixation. In: SLATTER, D.H. Textbook of small animal surgery. Philadelphia : Sauders, 1993. V.2. Cap.1641-1656.

GREEN, S.A. Complications of external skeletal fixation. Clinical Orthopaedics and Related Research, n.180, p.109$116,1983$.

HARARI, J. Techniques for application of metal external fixators. In: COMPLETE COURSE IN EXTERNAL SKeletal FIXATION, 5, 1996, Athens. Proceedings... Athens : Georgia Center for Continuing Education, 1996, 141p., p.10-16.

HULSE, D., HYMAN, W. Biomechanics of fracture fixation failure. The Veterinary Clinics of North America: Small Animal Practice, v.21, n.4, p.647-667, 1991.
JOHNSON, W.D., FISCHER, D.A. Skeletal stabilization with a multiplane external fixation device. Biomechanical evaluation and finite element model. Clinical Orthopaedics and Related Research, n.180, p.34-43, 1983.

KANEPS, A.J., SCHMOTZER,W.B. Fracture repair with transfixation pins and fiberglass cast in llamas and small ruminants. Journal the American Veterinary Medical Association, v.195, n.9, p.1257-1261,1989.

LEWIS, D.D., BLOOMBERG, M.S. Fijación óssea externa. Walthan Focus, v.4, n.4, p.2-18, 1994.

NUNAMAKER, D.M., NEWTON, C.D. Textbook of small animal orthopedics. Philadelphia : Lippincott, 1985. p.1123.

OBRASINSKI, E.B., PARDO, A.D., GRAEHLER, R. Biomechanical evaluation of acrilic external skeletal fixation in dogs and cats. Journal of American Veterinary Medical Association, v. 199, n.11, p.1590-1593, 1991.

OHASHI, T., INOUE, S., KAJIKAWA, K. External skeletal fixation using methilmethacrylate. Clinical Orthopaedics and Related Research, v.178, p.121-129, 1983.

PARDO, A.D. Relationship of tibial intramedulary pins to canine stifle joint structure: A comparison of normograde and retrogade insertion. Journal of the American Animal Hospital Association, v.30, Jul.-aug., p.369-374, 1994.

SCHRADER, S.C. Complications associated with the use of Steimann intramedulary pins and cerclage wires for fixation of long bone fractures. Veterinary Clinics of North America: Small Animal Practice, v.21, n.4, p.687-703, 1991.

SISK, T.D. External fixation: Historic review, advantages, disadvantages, complications, and indications. Clinical Orthopaedics and Related Research, v.180, n.15, p.15-22, 1983.

SMITH, M.M. Orthopedic infections. In: SLATTER, D.H. Textbook of small animal surgery. Philadelphia : Saunders, 1993. V.2. Cap.126, p.1685-1693.

SPIEGEL, P.G., VANDDERSCHILDEN, J.L. Minimal internal and external fixation in the treatment of open tibial fracture. Clinical Orthopedics and Related Research, v.178, p.96102, 1983.

TOOMBS, J.P. Nomenclature and instrumentation of external skeletal fixation systems. In: COMPLETE COURSE IN EXTERNAL SKELETAL FIXATION, 5, 1996, Athens: Proceedings... Athens : Georgia Center for Continuing Education, 1996. 141p. p.2-10.

TUDURY, E.A., RAISER, A.G. Redução de fraturas distais de fêmur de cães, empregando pinos de Steimann em substituição aos de Rush. Revista do Centro de Ciências Rurais, v.15, n.2, p.141-155, 1985.

WILLER, R.L., EGGER, E.L., HISTAND, M.B. Comparison of stainless steel versus acrylic for the connecting bar of external skeletal fixators. Journal of American Animal Hospital Association. v.27, p.541-548, 1991. 